\title{
IDENTIFICACIÓN DE PARÁSITOS GASTROINTESTINALES EN PRIMATES NO HUMANOS DEL ZOOLÓGICO PARQUE NATURAL DE PUCALLPA, PERÚ
}

\author{
Identification of Gastrointestinal Parasites in Nonhuman Primates of the \\ Pucalla Natural Zoological Park, Peru
}
Francesca Guerrero M. ${ }^{1}$, Enrique Serrano-Martínez ${ }^{1,3}$, Manuel Tantaleán V. ${ }^{1}$, Marco Quispe H. ${ }^{1}$, Gina Casas V. ${ }^{1}$

\section{RESUMEN}

El presente estudio determinó, mediante exámenes coprológicos, la presencia de parásitos gastrointestinales en primates no humanos en cautiverio del Zoológico Parque Natural de Pucallpa, ubicado en el departamento de Ucayali - Perú. Se recolectaron 72 muestras fecales colectivas de 58 primates de las especies Cebus apella (machín negro), Saguinus fuscicollis weddelli (pichico común o tití), Cebus albifrons (machín blanco), Lagothrix lagotricha (mono choro), Ateles paniscus chamek (maquisapa), Saimiri sciureus (frailecillo), Aotus nigriceps (musmuqui) y Alouatta seniculus (mono coto o aullador rojo). Las muestras fueron fijadas en formol al $10 \%$ a $60{ }^{\circ} \mathrm{C}$ y posteriormente analizadas mediante los métodos Directo, Ritchie, Sheather, Sedimentación y tinción de Ziehl-Neelsen. Los parásitos gastrointestinales hallados fueron Strongyloides cebus (56/72), Paratriotaenia oedipomidatis (8/72), Prosthernorchis elegans (7/72), Trichostrongylidae (6/72), Oxyuroideo (2/72), Entamoeba coli (2/72), quiste tipo coccidia (2/72), Crytosporidium spp (3/72) y Balantidium coli (3/72). De las 8 especies de primates estudiadas, todas presentaron al menos una especie de parásito gastrointestinal.

Palabras clave: parasitosis, gastrointestinal, coprológico, primates, cautiverio, Pucallpa, Perú

\section{Abstract}

The present study detected by stool examinations the presence of gastrointestinal parasites in nonhuman primates reared in captivity at the Pucallpa Natural Zoological Park, located in the department of Ucayali - Peru. A total of 72 collective fecal samples were collected from 58 individuals from species Cebus apella (brown capuchins), Saguinus fuscicollis weddelli (saddleback tamarins), Cebus albifrons (white-fronted capuchins), Lagothrix lagotricha (woolly monkey), Ateles paniscus chamek (spider monkey), Saimiri

\footnotetext{
${ }^{1}$ Grupo SALUVET-UPCH, Facultad de Veterinaria y Zootecnia, Universidad Peruana Cayetano Heredia, Lima

${ }^{2}$ E-mail: enrique.serrano@upch.pe
} 
sciureus (squirrel monkey), Aotus nigriceps (black-headed night monkey) and Alouatta seniculus (red howler monkey). Samples were fixed in $10 \%$ formalin at $60^{\circ} \mathrm{C}$ and analyzed by the direct method, and the Ritchie, Sheather, Sedimentation and Ziehl-Neelsen methods. Gastrointestinal parasites found were Strongyloides cebus (56/72), Paratriotaenia oedipomidatis (8/72), Prosthernorchis elegans (7/72), Trichostrongylidae (6/72), Oxyuroidea (2/72), Entamoeba coli (2/72), Coccidia oocysts (2/72), Cryptosporidium spp (3/72) and Balantidium coli (3/72). In the eight evaluated species, all had at least one parasite species.

Key words: parasites, gastrointestinal, stool, primates, captive, Pucallpa, Peru

\section{INTRODUCCIÓN}

En el Perú se conocen 39 especies de primates no humanos distribuidos en 12 géneros (Encarnación et al., 2000; Pacheco et al., 2009). Actualmente, los primates figuran entre los tipos de animales más cazados en la Amazonía Peruana, ya sea para su comercialización ilegal o como fuente de proteína animal para el consumo familiar (Aquino et al., 2000, 2007). La mayor incidencia de caza ocurre en primates no humanos de las especies Cebus apella, Alouatta seniculus y Lagothrix lagotricha (Aquino et al., 2000).

Además de la cacería clandestina y la destrucción del hábitat, las infecciones parasitarias juegan un rol importante en la capacidad de supervivencia de los primates, especialmente si se toma en cuenta que cada día existe mayor contacto entre primates y humanos, dándose el riesgo de transmisión de parásitos de humanos hacia los monos, especialmente de protozoarios como Balantidium coli, Entamoeba coli, E. histolytica y Giardia spp (Chinchilla et al., 2005; Figueiroa et al., 2001).

La presencia de parásitos intestinales se ha documentado en especies como Alouatta seniculus (mono aullador rojo o mono coto) en la Cuenca del Amazonas (Stoner et al., 2005), Aotus azarae y Aotus nancymaae en la Amazonía Peruana (Tantaleán et al.,
2005), Saguinus (Michaud et al. 2003; Phillips et al., 2004), Saimiri (Sarmiento et al., 1999; Arrojo, 2002; Michaud et al., 2003; Tantaleán et al., 2005) y Lagothrix lagotricha (Michaud et al., 2003). Del mismo modo, Phillips et al. (2004) reportaron la presencia de parásitos gastrointestinales en Alouatta seniculus, Aotus vociferans, Ateles bezlebuth chamek, Callicebus brunneus, Cebus albifrons, Cebus apella, Saguinus fuscicollis y Saimiri sciureus de la Reserva Nacional de Tambopata, Madre de Dios; y Carrasco et al. (2008) en Ateles belzebuth chamek del Parque Nacional del Manu.

En el Centro de Reproducción y Conservación de Primates del Proyecto Peruano de Primatología, IVITA, se clasificaron las causas de mortalidad en A. nancymae y A. vociferans menores de siete meses de edad, durante el periodo 1988-2002 ( $\mathrm{n}=1453)$, determinándose que $26 \%$ de las causas de mortalidad fue debida a procesos gastrointestinales (Sánchez et al., 2006).

El control parasitológico es un importante aspecto para el cuidado preventivo de la salud, particularmente en climas húmedos y calurosos. En tal sentido, determinar la presencia de parásitos gastrointestinales en primates no humanos en cautiverio permitirá tomar medidas preventivas y minimizar los efectos negativos de las infecciones parasitarias, además de mejorar la calidad de vida de estos animales. 


\section{Materiales y Métodos}

El estudio se llevó a cabo en el Zoológico Parque Natural de Pucallpa, departamento de Ucayali, Perú. La zona presenta un clima cálido y lluvioso, con temperatura promedio anual de $26^{\circ} \mathrm{C}\left(21-38^{\circ} \mathrm{C}\right)$, precipitación pluvial anual de $2344 \mathrm{~mm}$, humedad anual media de $84.2 \%$ y altitud de 154 msnm.

La población de primates no humanos era de 58 animales pertenecientes a ocho especies, con edades entre 6 meses a 9 años. Algunos de los animales del estudio fueron rescatados por la Dirección General Forestal y Fauna Silvestre de mercados locales y en otros casos fueron donados; por lo cual no contaban con registros de la procedencia pre captura. Los animales se encontraban en jaulas comunes por especie. Su alimentación era a base a frutas ad libitum y el agua de bebida procedía del río Ucayali.

En mayo de 2009, se recolectaron 72 muestras fecales colectivas de las ocho especies de primates no humanos (Cuadro 1) para determinar la presencia de endoparásitos. Las muestras se recogieron del suelo inmediatamente después que los individuos defecaron y se colocaron en frascos rotulados.

Las muestras de heces fueron divididas en dos partes: una fue colocada inmediatamente en frascos con formol al $10 \%$ y mantenidas a temperatura ambiente, para la detección de huevos y larvas de helmintos, y la otra parte fue mantenida en refrigeración para la detección de protozoarios. El tiempo promedio desde que se tomaron las muestras hasta que estas se analizaron en el laboratorio fue de tres días.

El procesamiento de las muestras se llevó a cabo en el Laboratorio de Parasitología de la Facultad de Veterinaria y Zootecnia de la Universidad Peruana Cayetano Heredia, siguiendo los protocolos del método directo, método de Ritchie, método de Sheather, téc- nica de Sedimentación y tinción Ziehl Neelsen. La identificación de las especies de parásitos fue llevada a cabo según los estudios realizados por Little (1966) y Tantaleán y Gozalo (1992, 1994).

Se determinó la presencia de parásitos gastrointestinales que afectan a los primates no humanos. Los resultados se expresaron en forma porcentual (Thrusfield, 1990).

\section{Resultados y Discusión}

La parasitosis gastrointestinal es una importante causa de morbilidad y mortalidad en primates del nuevo mundo, pudiendo provocarles daños mecánicos y procesos gastroentéricos, que lleven al animal a desbalances hídrico-electrolíticos y disminución en la absorción de nutrientes, con la consecuente pérdida de condición corporal; además, infestaciones masivas se pueden transformar en procesos obstructivos poniendo en riesgo la vida de los animales (Pérez et al., 2007).

Los primates son vulnerables a los efectos de las infecciones parasitarias debido a que generalmente viven en grupos sociales cerrados que facilitan su transmisión (Stoner et al., 2005). En este sentido, la prevalencia suele incrementarse en pequeñas áreas por la constante infección y reinfección como consecuencia de una mayor acumulación de parásitos (Müller, 2007); como ocurre en el Zoológico Parque Natural de Pucallpa, donde existe una alta densidad poblacional, que sumado a las pobres medidas de higiene, programas de desparasitación poco efectivos, nutrición inadecuada, estrés del cautiverio y condiciones climatológicas, entre otros, contribuyen a la presencia de parásitos.

En el presente estudio se identificaron nematodos (Strongyloides cebus, Trichostrongylidae, Oxyuroideo), cestodos (Paratriotaenia oedipomidatis), acantocéfalos (Prosthernorchis elegans) y protozoos 
Cuadro 1. Número de muestras colectivas de primates no humanos en cautiverio del Zoológico Parque Natural de Pucallpa

\begin{tabular}{lcc}
\hline Especie & N. de animales & $\begin{array}{c}\text { N. }{ }^{\circ} \text { de muestras } \\
\text { colectivas }\end{array}$ \\
\hline Cebus apella (machín negro) & 11 & 14 \\
Ateles paniscus chamek (maquisapa) & 13 & 10 \\
Saguinus fuscicollis weddelli (pichico) & 2 & 4 \\
Saimiri sciureus (mono fraile) & 7 & 8 \\
Cebus albifrons (machín blanco) & 7 & 7 \\
Lagothrix lagotricha (mono choro) & 12 & 18 \\
Aotus nigriceps (musmuqui) & 4 & 6 \\
Alouatta seniculus (mono coto o aullador rojo) & 2 & 5 \\
\hline Total & 58 & 72 \\
\hline
\end{tabular}

Cuadro 2. Presencia de parásitos protozoarios y cestodos gastrointestinales en primates no humanos criados en cautiverio en el Zoológico Parque Natural de Pucallpa (2009)

\begin{tabular}{|c|c|c|c|c|c|}
\hline \multirow[b]{2}{*}{ Primates } & \multicolumn{4}{|c|}{ Protozoo } & \multirow{2}{*}{$\begin{array}{c}\text { Cestodo } \\
\text { P. } \\
\text { oedipomidatis }\end{array}$} \\
\hline & E. coli & $\begin{array}{c}\text { Cryptosporidium } \\
\text { spp }\end{array}$ & $\begin{array}{l}\text { Quiste } \\
\text { Coccidia }\end{array}$ & B. coli & \\
\hline Ateles paniscus chamek & - & - & - & - & + \\
\hline $\begin{array}{l}\text { Saguinus fuscicollis } \\
\text { weddelli }\end{array}$ & + & - & - & - & - \\
\hline Saimiri sciureus & - & + & - & - & + \\
\hline Cebus apella & - & - & + & - & + \\
\hline Cebus albifrons & - & + & - & + & + \\
\hline Lagothrix lagotricha & + & - & + & - & + \\
\hline Aotus nigriceps & - & - & - & + & - \\
\hline Alouatta seniculus & - & - & - & - & - \\
\hline
\end{tabular}

(Entamoeba coli, ooquiste tipo coccidia, Crytosporidium spp y Balantidium coli) (Fig. 1), siendo el nematodo S. cebus el más frecuente. Al respecto, Toft y Eberhard (1998) sostienen que $S$. cebus es un parásito natu- ral en los monos de América Central y del Sur, reportándose su presencia en Saimiri, Lagothrix, Cebus, Ateles, Alouatta, Aotus y Saguinus (Gozalo et al., 1990; Nole, 2008; Stuart et al., 1990; Tantaleán et al., 1990; Karesh et al., 1998; Phillips et al., 2004). 
Cuadro 3. Presencia de parásitos nematodos y acantocéfalos gastrointestinales en primates no humanos criados en cautiverio en el Zoológico Parque Natural de Pucallpa (2009)

\begin{tabular}{|c|c|c|c|c|}
\hline \multirow{2}{*}{ Primates } & \multicolumn{3}{|c|}{ Nematodo } & \multirow{2}{*}{$\begin{array}{c}\text { Acantocéfalo } \\
\text { P. elegans }\end{array}$} \\
\hline & S. cebus & Trichostrongylidae & Oxyuroideo & \\
\hline Ateles pan iscus chamek & + & + & + & - \\
\hline Saguinus fuscicollis weddelli & + & - & - & - \\
\hline Saimiri sciureus & + & + & - & + \\
\hline Cebus apella & + & - & - & + \\
\hline Cebus albifrons & + & + & - & - \\
\hline Lagothrix lagotricha & + & - & - & + \\
\hline Aotus nigriceps & + & - & + & + \\
\hline Alouatta seniculus & + & - & - & - \\
\hline
\end{tabular}

La presencia de nematodos posiblemente se deba a su ciclo de vida directo, donde son transmitidos de animal a animal por contaminación fecal o contacto directo, ya sea por ingestión de huevos, penetración por la piel y transmisión lactogénica o intrauterina (Toft y Eberhard, 1998; Müller, 2007).

Respecto a los cestodos, Paratriotaenia oedipomidatis fue la segunda especie más frecuentemente observada, encontrándose en A. paniscus chamek, S. sciureus, C. apella, C. albifrons y L. lagotricha (Cuadro 2). Este parásito ha sido reportado en primates no humanos como Saguinus spp, Callithrix spp y Aotus (Tantaleán y Gozalo, 1994; Müller, 2007).

En siete muestras de $S$. scureus, $C$. apella, L. lagotricha y A. nigriceps se identificó una sola especie de acantocéfalo, $P$. elegans. Este parásito ha sido reportado en Saguinus, Saimiri, Alouatta, Aotus, Ateles, Cebus, Cebuella, Lagothrix y Callicebus
(Horna y Tantaleán, 1983; Tantaleán y Gozalo, 1994; Arrojo, 2002; Michaud et al., 2003; Phillips et al., 2004; Stoner et al., 2005; Tantaleán et al., 2005). Pérez et al. (2007) indican que la presencia del $P$. elegans es particularmente grave por efecto del estrés durante el cautiverio, presencia permanente del principal huésped intermediario en los sitios de alojamiento y por la pobre respuesta a los tratamientos médicos.

De las cuatro especies de protozoos hallados, Entamoeba coli procede del humano, lo cual sugiere una antropozoonosis, más aun, teniendo en cuenta que varios de estos animales fueron decomisados de mercados ilegales o donados por personas que los habían tenido cautivos por periodos variados. $\mathrm{Al}$ respecto, Phillips et al. (2004), trabajando con A. paniscus chamek de la Reserva Nacional de Tambopata, reportaron la presencia de tres protozoos (Chilomastix spp, Blastocystis spp e Iodamoeba spp), los cuales están ligados a influencia antropogénica (Stuart et al., 1990; Chinchilla et al., 2005). 


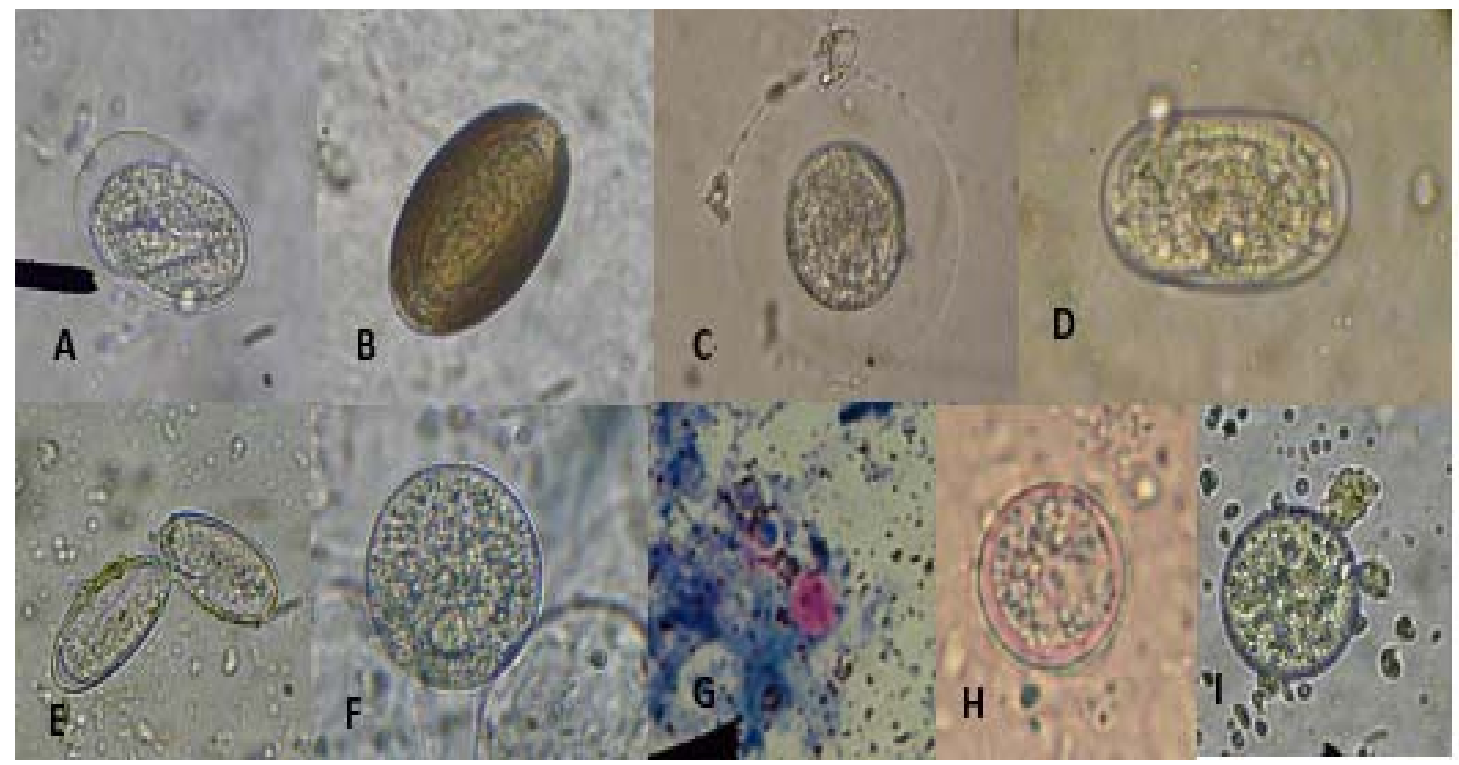

Figura 1. Parásitos gastrointestinales en primates no humanos criados en cautiverio en el Zoológico Parque Natural de Pucallpa (2009). A. Huevo de Strongyloides cebus (40x). B. Huevo de Prosthenorchis elegans (40x). C. Huevo de Paratriotaenia oedipomidatis (10x). D. Huevo Trichostrongylidae (40x). E. Huevo Oxyuroideo (40x). F. Quiste de Balantidium coli (40x) encontrado en Cebus albifrons. G. Ooquiste de Cryptosporidium spp (100x). H. Quistes de Entamoeba coli (40x) hallado en Saguinus fuscicollis weddelli. I. Ooquiste de coccidia (40x)

Asimismo, en el Perú se ha demostrado la presencia de protozoarios intestinales de los géneros Blastocystis, Chilomastix, Endolimax, Entamoeba, Iodamoeba, Giardia, Cryptosporidium, Isospora y Balantidium en Alouatta, Ateles, Cebus, Saimiri, Saguinus y Aotus (Tantaleán y Gozalo, 1994; Gozalo y Tantaleán, 1996; Phillips et al. 2004).

Especímenes de las ocho especies de primates no humanos en estudio presentaron al menos una especie de parásito, como es el caso de Alouatta seniculus, que presentó una especie de nematodo (S. cebus). Otras especies como S. sciureus, C. albifrons y L. lagotricha presentaron mayor variedad (5) de parásitos gastrointestinales (Cuadros 2 y 3).

La infección parasitaria en humanos puede ser un riesgo para la salud de los primates no humanos, ya que estos animales son comúnmente mantenidos como mascotas o criados para el consumo humano en áreas rurales, pudiendo los parásitos ser potencialmente fuentes de antropozoonosis como es el caso de Ascaris lumbricoides, Trichuris trichiura, Hymenolepis nana, Strongyloides stercoralis y Ancylostoma spp, entre otros (Michaud et al., 2003).

\section{ConClusiones}

- Se reporta la presencia de nematodos (Strongyloides cebus, Trichostrongylidae, Oxyuroideo), cestodos (Paratriotaenia oedipomidatis), acantocéfalos (Prosthernorchis elegans) y protozoos (Entamoeba coli, quiste tipo coccidia, Crytosporidium spp y Balan-tidium coli) en primates no humanos del Zoológico Parque Natural de Pucallpa. 
- Las especies S. sciureus, C. albifrons y L. lagotricha presentaron hasta cinco especies de parásitos gastrointestinales; asimismo, especímenes de todas las especies en estudio presentaron al menos una especie de parásito.

\section{Agradecimiento}

Los autores agradecen al Sr. Elciario Naranjo, Gerente del Zoológico Parque Natural de Pucallpa, por el apoyo brindado en campo durante la colección de muestras y ejecución del estudio.

\section{Literatura Citada}

1. Aquino R, Bodmer RE, Gil JG. 2000. Impacto de la caza en poblaciones de primates de la cuenca del río Samiria, Reserva Nacional Pacaya-Samiria. La primatología en el Perú. Vol II. Lima: UNMSM. p 81-91.

2. Aquino $R$, Terrones $C$, Navarro $E$, Terrones $W$. 2007. Evaluación del impacto de la caza en mamíferos de la cuenca del río Alto Itaya, Amazonía Peruana. Rev Perú Biol 14: 181-186.

3. Arrojo L. 2002. Parásitos de animales silvestres en cautiverio en Lima, Perú. Rev Perú Biol 9: 118-120.

4. Carrasco F, Tantaleán M, Gibson KN, Gibson N. 2008. Prevalencia de helmintos intestinales de una población de monos maquisapas silvestres Ateles belzebuth chamek en el Parque Nacional de Manu - Perú. Neotrop Helminthol 2: 19-26.

5. Chinchilla M, Guerrero O, Gutiérrez GA, Sánchez R, Rodríguez B. 2005. Parásitos intestinales en monos congo Alouatta palliata de Costa Rica. Rev Biol Trop 53: 437-445.

6. Encarnación F, Moya L, Aquino R, Tapia J, Soini P. 2000. Situación y estado actual de las especies de primates no humanos en el Perú. En: La primatología en el Perú. Vol II. Lima: UNMSM. p 219-228.
7. Figueiroa $M$, Bianques $J$, Dowell $M$, Alves R, Evecio A. 2001. Perfil coproparasitológico de mamíferos silvestres en cautiverio en el estado de Pernambuco, Brasil. Parasitol 25: 3-4.

8. Gozalo A, Aquino R, Montoya E. 1990. Prevalencia de parásitos gastrointes-tinales en Aotus nancymai capturados en la Amazonía Peruana. La primatología en el Perú. Vol I. Lima: UNMSM. p 585-587.

9. Gozalo A, Tantaleán M. 1996. Parasitic protozoa in neotropical primates. Lab Primate Newsl 35(3): 1-7.

10. Horna M, Tantaleán M. 1983. Parásitos de primates peruanos: Helmintos del mono fraile y del pichico barba blanca. Bol Lima 27: 54-58.

11. Karesh WB, Wallance RB, Painter RLE, Rumiz D, Braselton WE, Dierenfeld ES, Puche H. 1998. Immobilization and health assessment of freeranging black spider monkeys (Ateles paniscus chamek). J Primatol 44: 107123.

12. Little MD. 1966. Comparative morphology of six species of Strongyloides (Nematoda) and redefinition of the genus. J Parasitol 52: 69-84.

13. Michaud C, Tantaleán M, Ique C, Montoya E, Gonzalo A. 2003. A survey for helminth parasites in feral New World non-human primate populations and its comparison with parasitological data from man in the region Iquitos - Peru. J Med Primatol 32: 341-345.

14. Müller B. 2007. Determinants of the diversity of intestinal parasite communities in sympatric New World Primates (Saguinus mystax, Saguinus fuscicollis, Callicebus cupreus). PhD Thesis. Germany: Tierärztliche Hochschule Hannover. 217 p.

15. Nole I. 2008. Parasitismo gastrointestinal en el mono tocón moreno (Callicebus brunneus) que habita en bosques con diferente grado de perturbación antropogénica en el departamento de Madre de Dios. Tesis de Médico Veterinario. Lima: Univ Nac Mayor de San Marcos. 46 p. 
16. Pacheco V, Cadenillas $R$, Salas E, Tello C, Zeballos H. 2009. Diversidad y endemismo de los mamíferos del Perú. Rev Peru Biol 16: 5-32.

17. Pérez J, Ramírez D, Hernández CA. 2007. Prosthenorchis sp en titíes grises (Saguinus leucopus). Rev CES Med Vet Zootec 2(1): 51-57.

18. Phillips K, Haas MM, Grafton B, Yrivarren M. 2004. Survey of the gastrointestinal parasites of the primate community at Tambopata Nacional Reserve, Peru. J Zool 264: 149-151.

19. Sánchez $N$, Gálvez H, Montoya $E$, Gozalo A. 2006. Mortalidad en crías de Aotus sp. (primates: cebidae) en cautiverio: Una limitante para estudios biomédicos con modelos animales. Rev Peru Med Exp Salud Pública 23: 221-224.

20. Sarmiento L, Tantaleán M, Huiza A. 1999. Nematodos parásitos del hombre y de los animales en el Perú. Rev Per Parasitol 14: 9-65.

21. Stoner KE, González Di Pierro AM, Maldonado S. 2005. Infección de parásitos intestinales de primates: Implicaciones para la conservación. Univ Ciencia (Nro. Especial) 2: 61-72.
22. Stuart MD, Greenspan LL, Glander KE, Clarke MR. 1990. Coprological survey of parasites of wild mantled howling monkeys, Alouatta palliata palliata. J Wildlife Dis 26: 547-549.

23. Tantaleán M, Gonzalo A, Montoya E. 1990. Notes on some helminth parasites from Peruvian monkeys. Lab Primate News 29: 6-8.

24. Tantaleán M, Gozalo A. 1994. Parasites of the Aotus monkey. In: Baer JF, Weller RE, Kakoma I (eds). Aotus: The Owl Monkey. San Diego, USA: Academic Press. p 353-374.

25. Tantaleán M, Sánchez L, Gómez L, Huiza A. 2005. Acantocéfalos del Perú. Rev Peru Biol 12: 83-92.

26. Tantaleán M, Gozalo A. 1992. Ascariasis en primates no humanos del Perú. Rev Per Med Trop UNMSM 6: 87.

27. Thrusfield M. 1990. Epidemiología veterinaria. España: Acribia. p. 228-230.

28. Toft JD, Eberhard ML. 1998. Parasitic diseases. In: Bennet BT, Abee CR, Henrickson R (eds). Nonhuman primates in biomedical research. San Diego, USA: Academic Press. p 111-205. 